USITP 2001-8

solv-int/0110021

\title{
A unified treatment of quartic invariants at fixed and arbitrary energy.
}

\author{
Max Karlovini* \\ Department of Physics - Stockholm University \\ Giuseppe Pucacco ${ }^{\dagger}$ \\ Dipartimento di Fisica - Università di Roma "Tor Vergata" \\ Kjell Rosquist ${ }^{\ddagger}$ and Lars Samuelsson ${ }^{\S}$ \\ Department of Physics - Stockholm University
}

\begin{abstract}
Two-dimensional Hamiltonian systems admitting second invariants which are quartic in the momenta are investigated using the Jacobi geometrization of the dynamics. This approach allows for a unified treatment of invariants at both arbitrary and fixed energy. In the differential geometric picture, the quartic invariant corresponds to the existence of a fourth rank Killing tensor. Expressing the Jacobi metric in terms of a Kähler potential, the integrability condition for the existence of the Killing tensor at fixed energy is a non-linear equation involving the Kähler potential. At arbitrary energy, further conditions must be imposed which lead to an overdetermined system with isolated solutions. We obtain several new integrable and superintegrable systems in addition to all previously known examples.
\end{abstract}

PACS numbers: $03.20 .+\mathrm{i} \quad 11.30 .-\mathrm{j}$

*e-mail: max@physto.se

†e-mail: pucacco@roma2.infn.it

‡e-mail: kr@physto.se

$\S$ e-mail: larsam@physto.se 


\section{Introduction}

The study of Hamiltonian systems by means of geometric techniques provides fruitful clues about their integrability [1, 2, 3]. In particular, geometrization of the dynamics by using the Jacobi metric [4, 胢, is a standard tool to turn a natural Hamiltonian system into a geodesic flow over a suitable Riemannian manifold. Therefore, it is natural to investigate integrability of two-dimensional systems by looking for invariants corresponding to Killing tensors of a conformal Riemannian geometry. These geometric objects in fact directly produce invariants polynomial in the momenta: the degree of this polynomial is the same as the rank of the Killing tensor. Since the energy enters in the Jacobi metric as a parameter, the conditions for the existence of a Killing tensor may happen to be satisfied only at a fixed value of energy. In this case we speak of integrability at fixed energy in distinction to the usual notion of integrability which is understood to be valid at arbitrary energy.

In [2], quadratic invariants at arbitrary and fixed energy (respectively, strongly and weakly conserved phase-space functions) for 2-dimensional Hamiltonian systems were treated in a unified way. The integrability condition for quadratic invariants involves an arbitrary analytic function $S(z)$. For invariants at arbitrary energy, the function $S(z)$ is a second degree polynomial with real second derivative and the integrability condition then reduces to the classical Darboux's condition for quadratic invariants at arbitrary energy [6]. Thereafter, the possibility of searching for linear and quadratic invariants at fixed energy was addressed and some examples of system admitting a second quadratic invariant at zero energy were provided.

Weak invariants, also called configurational invariants, have been discussed by Hall [7] and by Sarlet, Leach and Cantrijn 88. Hietarinta [9], in his account of the direct methods for the search of the second invariant, also provides a review of all the known systems admitting one or more configurational invariants. Generalizing the approach of paper [2], Karlovini and Rosquist [10] have discussed the existence of invariants cubic in the momenta at both fixed and arbitrary energy. Beside giving a list of all known systems admitting a cubic strong invariant, they also find a superintegrable system admitting a cubic invariant related to an energy dependent linear invariant. In the present paper we are going to discuss the case of the quartic invariant associated with the existence of a fourth-rank Killing tensor. Fourth-rank Killing tensors have previously been studied by one of the authors (see [11]) in the Lorentzian case.

In analogy to the third-rank case, it turns out that it is natural to introduce a Kähler potential for the Jacobi metric. Expressing the conditions imposed on the geometry by Killing tensor equations in terms of this Kähler potential, there remains a system of nonlinear partial differential equations. Imposing the condition of strong integrability, it turns out that in general this system becomes overdetermined, so that, like for cubic invariants, only isolated mechanical systems exist with a second invariant quartic in the momenta. Due to the complexity of the system of equations, it is impossible to get a fully general solution. However, with our approach we are able to find some new integrable and superintegrable cases. In particular, one may wonder if the knowledge of a weak invariant can provide information about the global dynamical behaviour of the system. We introduce a method which, starting from a family of weakly integrable systems, leads to finding a higher-order invariant for isolated members of the family which are therefore strongly integrable. The second invariant depends on the energy as a parameter.

The plan of the paper is as follows: in section 2 we recall the necessary and sufficient conditions for the existence of a Killing tensor in the Jacobi geometry and their application in problems of analytical mechanics; in section 3 we examine the particular case of fourth-rank Killing tensors; in section 4 we analize the conditions for strong integrability with a quartic invariant; in section 5 we present the method by which, starting from a family of weakly integrable systems, isolated examples of strongly integrable systems with quartic second invariant can be found; in section 6 we give tables of integrable systems admitting a quartic invariant which include new integrable and superintegrable systems in addition to all known cases; in section 7 we give our conclusions.

\section{Jacobi geometry and Killing tensors}




\subsection{Geometric representation of the dynamics}

We are interested in the classical two-dimensional systems with Hamiltonian function

$$
\mathcal{H}=\frac{1}{2}\left(p_{x}^{2}+p_{y}^{2}\right)+V(x, y)
$$

The approach followed in studying the integrability properties of these systems is based on the Jacobi geometrization procedure, which associates to the Hamiltonian flow produced by (11), a geodesic flow on a Riemannian manifold endowed with a positive definite metric

$$
d s_{J}^{2}=g_{\alpha \beta} d q^{\alpha} d q^{\beta}, \quad \alpha, \beta=1,2 .
$$

It can be shown (see, e.g. [4]), that the Jacobi metric

$$
g_{\alpha \beta}=2(E-V) h_{\alpha \beta},
$$

where $h_{\alpha \beta}$ is the metric of the flat space, generates a geodesic flow corresponding to the natural mechanical system with potential $V=V(q)$ at energy $E$. In fact, introducing the geodesic, or "Jacobi", Hamiltonian

$$
\mathcal{H}_{J}=\frac{1}{2} \frac{1}{2(E-V)} h^{\alpha \beta} p_{\alpha} p_{\beta} \equiv \frac{1}{2},
$$

the geodesic equations

$$
\begin{aligned}
& \frac{d q^{\alpha}}{d s_{J}}=\frac{\partial \mathcal{H}_{J}}{\partial p_{\alpha}}=\frac{1}{2(E-V)} h^{\alpha \beta} p_{\beta}, \\
& \frac{d p_{\alpha}}{d s_{J}}=-\frac{\partial \mathcal{H}_{J}}{\partial q^{\alpha}}=-\frac{1}{2(E-V)} \frac{\partial V}{\partial q^{\alpha}},
\end{aligned}
$$

are equivalent to the canonical equations of motion given by the Hamiltonian (1),

$$
\begin{aligned}
\frac{d q^{\alpha}}{d t} & =\frac{\partial \mathcal{H}}{\partial p_{\alpha}}, \\
\frac{d p_{\alpha}}{d t} & =-\frac{\partial \mathcal{H}}{\partial q^{\alpha}},
\end{aligned}
$$

if the natural identification of canonical coordinates

$$
q^{1}=x, q^{2}=y, \quad p_{1}=p_{x}, \quad p_{2}=p_{y}
$$

is made. The phase-space trajectories are parametrized by the affine parameter $s_{J}$ that is related to the standard time variable by

$$
d s_{J}=2[E-V(q(t))] d t
$$

The Jacobi geometry corresponding to Hamiltonian (11) is then

$$
d s^{2}=2 G\left(d x^{2}+d y^{2}\right)=2 G d z d \bar{z}, \quad G=E-V,
$$

where we have introduced null variables $z=x+i y$ and $\bar{z}=x-i y$.

All tensor calculations will be done in the standard null frame defined as

$$
\Omega^{0}=\sqrt{G} d z, \quad \Omega^{\overline{0}}=\sqrt{G} d \bar{z}
$$

in which the metric takes the simplest possible form

$$
d s^{2}=2 d \Omega^{0} d \Omega^{\overline{0}}
$$

We use the convention that tensor indices in this frame take the values 0 and $\overline{0}$, while in any coordinate frame the values will be the names of the coordinates (e.g. $z$ and $\bar{z}$ ). 


\subsection{Invariants polynomial in the momenta and Killing tensors}

One of the standard tools of the geometric approach is the investigation of integrability by looking for invariants generated by Killing tensors. Let us see the simple case of second-order Killing tensor equations

$$
K_{(\alpha \beta ; \gamma)}=0 .
$$

The existence of a second-rank Killing tensor, that is a symmetric tensor which satisfies eq.(12), implies the existence of a conserved quadratic function

$$
I_{J}=K^{\alpha \beta} p_{\alpha} p_{\beta}
$$

commuting with the Jacobi Hamiltonian. In fact, it is easy to check that the vanishing of the Poisson bracket of this function with the Hamiltonian implies eq.(12). In analogy with the theory of second order invariants, the Jacobi geometry approach leads, as it stands, to the determination of higher-order invariants. Generalizing eq.(12), an $m^{\text {'th }}$ rank Killing tensor is a symmetric tensor satisfying equation

$$
K_{\left(\mu_{1} \mu_{2} \ldots \mu_{m} ; \mu_{m+1}\right)}=0 .
$$

It gives rise to the $m^{\prime}$ 'th order (in the momenta) invariant

$$
I_{J}=K^{\mu_{1} \mu_{2} \ldots \mu_{m}} p_{\mu_{1} \ldots p_{\mu_{m}}} .
$$

Two remarks are in order here:

1) The function (15) is a weak invariant, in the sense that, in general, it is a conserved quantity in the dynamics fixed by the given value of the energy appearing in the definition of the Jacobi geometry (9). To grant it the full title of strong invariant, it must satisfy the additional requirement of satisfying the Killing tensor equations at arbitrary energy.

2) The function (15), let us call it the Jacobi invariant, is a homogeneous polynomial of degree equal to the rank of the corresponding Killing tensor. To transform it into the ordinary invariant in the physical time gauge there is a straightforward recipe consisting in replacing the parameter $E$ appearing in $I_{J}$ with the corresponding Hamiltonian function (11). As a consequence, the physical invariant

$$
I=\left.I_{J}\right|_{E \rightarrow \mathcal{H}}
$$

becomes a polynomial which is either even or odd in the momenta.

Karlovini and Rosquist (2000, [10]) have discussed the existence of third-rank Killing tensors, giving a list of all known integrable or superintegrable systems admitting a cubic strong invariant. Here we are going to discuss the case of the quartic invariant associated with the existence of a fourth-rank Killing tensor. Moreover, we want to exploit the results concerning the existence of higher-order invariants to generate new solutions starting from the assumption of the existence of a second-rank Killing tensor.

\section{Quartic invariants corresponding to fourth rank Killing tensors}

In this section we derive the necessary and sufficient integrability condition for the Jacobi metric to admit a fourth-rank Killing tensor at a fixed value of the energy parameter $E$. In the following section we proceed by finding the conditions that ensure that the Killing tensor equations be satisfied at arbitrary values of the energy, with the restriction to the case in which the energy dependence of the Killing tensor is such that the corresponding invariant of the physical Hamiltonian is quartic in the momenta. As usual in our approach (see [11]), we decompose the fourth-rank Killing tensor in the form:

$$
K_{\alpha \beta \gamma \delta}=P_{\alpha \beta \gamma \delta}+P_{(\alpha \beta} g_{\gamma \delta)}+\frac{3}{8} K g_{(\alpha \beta} g_{\gamma \delta)}
$$

or equivalently

$$
K_{\alpha \beta \gamma \delta}=P_{\alpha \beta \gamma \delta}+K_{(\alpha \beta} g_{\gamma \delta)}-\frac{1}{8} K g_{(\alpha \beta} g_{\gamma \delta)}
$$


where $K_{\alpha \beta}=K^{\gamma}{ }_{\alpha \beta \gamma}$ and $K=K_{\gamma}^{\gamma} . P_{\alpha \beta \gamma \delta}$ is the trace-free (conformal) part, $P_{\alpha \beta}=P^{\gamma}{ }_{\alpha \beta \gamma}$ its trace and $K$ the full trace of the Killing tensor. In this way, the Killing tensor equations are split into the trace-free "components" and the equation for the trace

$$
\begin{gathered}
P_{(\alpha \beta \gamma \delta ; \mu)}-\frac{1}{2} P_{(\alpha \beta \gamma ; \nu}^{\nu} g_{\delta \mu)}=0 \\
P_{(\alpha \beta ; \gamma)}-\frac{1}{2} P_{(\alpha ; \nu}^{\nu} g_{\gamma \delta)}+\frac{1}{2} P^{\nu}{ }_{\alpha \beta \gamma ; \nu}=0 \\
K_{, \alpha}=-\frac{4}{3} P^{\beta}{ }_{\alpha ; \beta} .
\end{gathered}
$$

It is advantageous to employ also the coordinate frame components of the conformal part and of its trace when parametrizing the five independent components of $K_{\alpha \beta \gamma \delta}$. Thus, the components of the Killing tensor can be written as

$$
\begin{aligned}
K_{0000} & =G^{2} P^{\bar{z} \bar{z} \bar{z}}=G^{2} \bar{S} \\
K_{000 \overline{0}} & =\frac{1}{2} P_{00}=\frac{1}{2} G P^{\bar{z} \bar{z}}=\frac{1}{2} G \overline{R(z, \bar{z})} \\
K_{00 \overline{0} \overline{0}} & =\frac{1}{4} K \\
K_{0 \overline{0} \overline{0} \overline{0}} & =\frac{1}{2} P_{\overline{0} \overline{0}}=\frac{1}{2} G P^{z z}=\frac{1}{2} G R(z, \bar{z}) \\
K_{\overline{0} \overline{0} \overline{0} \overline{0}} & =G^{2} P^{z z z z}=G^{2} S,
\end{aligned}
$$

where, as usual, $S=S(z)$ is a holomorphic function, so that $\bar{S}=\bar{S}(\bar{z})$ comes from $(19)$, but the function $R=R(z, \bar{z})$ is a generic function of the arguments. With this parametrization, the equation for the trace (21) has the integrability condition

$$
2\left(G_{, z z} R-G_{, \bar{z} \bar{z}} \bar{R}\right)+3\left(G_{, z} R_{, z}-G_{, \bar{z}} \bar{R}_{, \bar{z}}\right)+G\left(R_{, z z}-\bar{R}_{, \bar{z} \bar{z}}\right)=0,
$$

which is analogous to that obtained for the second rank case (but now $R$ is not holomorphic!).

We now proceed to solve the Killing tensor equations. Eq.(20) gives

$$
S^{-3 / 4} R_{, \bar{z}}+2\left(S^{1 / 4} G\right)_{, z}=0
$$

and its complex conjugate. In analogy with the approach followed in [2, 10], we make a coordinate transformation to put the conformal Killing tensor in the simplest (standard) form. Using a conformal transformation of the form

$$
\begin{aligned}
& w=H(z), \\
& w=X+i Y, \\
& z=x+i y,
\end{aligned}
$$

the transformation of the conformal tensor is such that

$$
\tilde{S}(w):=P^{w w w w}=\left[H^{\prime}(z)\right]^{4} P^{z z z z}=\left[H^{\prime}(z)\right]^{4} S(z) .
$$

If we make the standard choice

$$
\tilde{S}(w):=P^{w w w w}=1
$$

the conformal transformation is then provided by the function

$$
H^{\prime}(z)=[S(z)]^{-1 / 4} .
$$

Eq.(24) becomes

$$
\left(S^{-1 / 2} R\right)_{, \bar{w}}+2 \tilde{G}_{, w}=0,
$$


with

$$
\tilde{G}=\left|H^{\prime}(z)\right|^{-2} G=\sqrt{|S|} G .
$$

Moreover, under the conformal transformation generated by eq.(28), the function $R$ transforms as

$$
\tilde{R}=P^{w w}=\left[H^{\prime}(z)\right]^{2} P^{z z}=S^{-1 / 2} R
$$

Inserting $\tilde{R}$ into eq.(29), we have the set

$$
\begin{aligned}
& \tilde{R}_{, \bar{w}}+2 \tilde{G}_{, w}=0, \\
& \tilde{\tilde{R}}_{, w}+2 \tilde{G}_{, \bar{w}}=0 .
\end{aligned}
$$

The solution of this system can be found in terms of a real Kähler potential $\mathcal{K}(w, \bar{w})$ :

$$
\begin{aligned}
\tilde{G} & =\mathcal{K}_{, w \bar{w}}, \\
\tilde{R} & =-2 \mathcal{K}_{, w w} .
\end{aligned}
$$

whereas the integrability condition (23) determines the following equation for the Kähler potential:

$$
\Im\left\{\left(\mathcal{K}_{, w w w} \mathcal{K}_{, w \bar{w}}+2 \mathcal{K}_{, w w \bar{w}} \mathcal{K}_{, w w}\right)_{, w}\right\}=0 .
$$

This is the necessary and sufficient condition for the existence of a fourth rank Killing tensor. In analogy to the third rank case (see [10]) and in contrast to the first and second rank case [2], the condition is highly nonlinear. The same condition has been already found in [7] and treated in [9], even if not in the context of the present geometric approach. Note that in [9], eq.(7.5.15), there is a misprint for a factor 2 missing.

In the standardized coordinate frame the second invariant can be written in the form

$$
I_{J}=2 \Re\left\{p_{w}^{4}+2 \tilde{R} \mathcal{H}_{J} p_{w}^{2}\right\}+\frac{3}{2} \mathcal{H}_{J}^{2} K
$$

whereas in the original null coordinate frame the second invariant is

$$
I_{J}=2 \Re\left\{S p_{z}^{4}+2 R \mathcal{H}_{J} p_{z}^{2}\right\}+\frac{3}{2} \mathcal{H}_{J}^{2} K,
$$

where

$$
\mathcal{H}_{J}=\frac{1}{G} p_{z} p_{\bar{z}}=\frac{1}{\tilde{G}} p_{w} p_{\bar{w}}=p_{0} p_{\overline{0}} \equiv \frac{1}{2},
$$

is the Jacobi Hamiltonian expressed in the three different reference frames. The trace function $K$ is found by integrating the system

$$
\begin{aligned}
& \frac{3}{4} K_{, \bar{w}}+\tilde{R}_{, w} \tilde{G}+2 \tilde{R} \tilde{G}_{, w}=0, \\
& \frac{3}{4} K_{, w}+\overline{\tilde{R}}_{, \bar{w}} \tilde{G}+2 \overline{\tilde{R}} \tilde{G}_{, \bar{w}}=0 .
\end{aligned}
$$

\section{Arbitrary energy invariants}

Eq.(34) gives the the necessary and sufficient condition for a two-dimensional Riemannian geometry to admit a fourth-rank Killing tensor. If we interpret the geometry according to eq.(9) as that providing a natural mechanical system, we can derive the additional conditions that make the Killing tensor equations satisfied for every value of the energy $E$. We use the following form for the Kähler potential,

$$
\mathcal{K}=E[z \bar{z}+2 \Re\{\Lambda(z)\}]-\Psi,
$$

where $\Lambda$ is a holomorphic function independent of $E$ and the real pre-potential $\Psi$ is such that

$$
\Psi_{, z \bar{z}}=V
$$


In fact, no loss of generality is implied by letting the energy dependence of the Kähler potential be prescribed by eq.(39) and by taking the function $S_{4}(z)$ to be energy independent, given that we only take interest in the cases for which the physical invariant $I$ is a quartic polynomial in the momenta. Since the analogous statement was made without proving it for the cubic case 10], we give a proof here. We begin by noting that the physical invariant $I$, obtained from the Jacobi invariant $I_{J}$ of eq. (36) according to prescription (16), can be written as

$$
I=2 \Re\left\{S p_{z}^{4}+B p_{z}^{3} p_{\bar{z}}\right\}+C\left(p_{z} p_{\bar{z}}\right)^{2},
$$

where

$$
\begin{aligned}
B & =2 R G^{-1}, \\
C & =\frac{3}{2} K G^{-2} .
\end{aligned}
$$

Since the energy parameter $E$ is now assumed to have been replaced by the physical Hamiltonian $\mathcal{H}$, the functions $S, B$ and $C$ will be dependent on the momenta $p_{z}$ and $p_{\bar{z}}$, but clearly only through the combination $p_{z} p_{\bar{z}}$ that appears in $\mathcal{H}$. This implies that all five terms on the right hand side of eq. (41) have to be quartic polynomials in the momenta since $I$ is quartic by definition. This can be clearly seen by viewing $I$ as a function of the two independent momenta functions $p_{z} p_{\bar{z}}$ and $p_{z} / p_{\bar{z}}$, rather than the momenta $p_{z}$ and $p_{\bar{z}}$ themselves. Indeed, $I$ can be recast into the form

$$
I=\left(p_{z} p_{\bar{z}}\right)^{2} \sum_{k=-2}^{2} Q_{k}\left(p_{z} / p_{\bar{z}}\right)^{k},
$$

where

$$
\begin{aligned}
& Q_{-2}=\overline{Q_{2}}=S, \\
& Q_{-1}=\overline{Q_{1}}=B, \\
& Q_{0}=C .
\end{aligned}
$$

Clearly, with the coefficients of $\left(p_{z} / p_{\bar{z}}\right)^{k}$ only depending on the momenta through $p_{z} p_{\bar{z}}$, there can be no cancellation of possible non-quartic polynomial dependence in the individual terms. Hence, we conclude that the functions $S p_{z}^{4}, B p_{z}^{4} p_{\bar{z}}$ and $C\left(p_{z} p_{\bar{z}}\right)^{2}$, as well as the complex conjugates of the first two, must be quartic momenta polynomials. It immediately follows that $S, B$ and $C$ are polynomials in $\left(p_{z} p_{\bar{z}}\right)^{-1}$, with coefficients depending only on $z$ and $\bar{z}$, of degree zero, one and two, respectively. This directly proves the part of statement about $S$ having no energy dependence. Moreover, since $G=E-V$ turns into $T=2 p_{z} p_{\bar{z}}$ when $E$ is replaced by $\mathcal{H}=T+V$, it now follows from eqs.(42) that the functions $R$ and $K$ are restricted to be first respectively second degree polynomials in $p_{z} p_{\bar{z}}$, which is the same as saying that they must be the same type of polynomials in the energy parameter $E$, before making the substitution $E \rightarrow \mathcal{H}$. Using the second of eqs.(33) and the conformal transformation formulae given by eqs.(28) and (31), the function $R$ can be expressed in terms of $S$ and $\mathcal{K}$ according to

$$
R=-2\left(S \mathcal{K}_{, z z}+\frac{1}{4} S^{\prime} \mathcal{K}_{, z}\right)
$$

while eqs.(38), which notably are form invariant under conformal transformations, give that the trace $K$ is given by integrating

$$
\frac{3}{4} K_{, \bar{z}}+R_{, z} G+2 R G_{, z}=0
$$

and its complex conjugate. Now, there is clearly no loss in generality to write the Kähler potential $\mathcal{K}$ as

$$
\mathcal{K}=E[z \bar{z}+2 \Re\{\Lambda(E, z)\}]-\Psi(z, \bar{z}),
$$

with $\Lambda$ being analytic in $z$ and $\Psi$ being energy independent. Moreover, since $S$ is energy independent and $R$ is a first degree polynomial in $E$, it follows from eq. (45) that we are able to write $\Lambda(E, z)$ as

$$
\Lambda(E, z)=\Lambda_{0}(z)+\Lambda_{1}(E, z),
$$


where $\Lambda_{1}(E, z)$ does not contribute to eq.(45). However, since eq.(46) shows that $\Lambda(z)$ enters into $K_{, \bar{z}}$ only through $R$, we might as well set $\Lambda_{1}(E, z)$ to zero, which proves the fact that we could have taken $\Lambda(E, z)$ to be energy independent from the outset. We may finally note that with both $R$ and $G$ being first degree polynomials in $E$, we find from eq.(46) that $K_{, \bar{z}}$ is a second degree polynomial in $E$, which indeed is a necessary and sufficient condition for $K$ to be a second degree polynomial as well, up to addition of an irrelevant integration constant which in principle can have an arbitrary energy dependence. This completes the proof that the form of the Kähler potential given by eq.(39) is the most general one which is needed for full generality.

We are now in the position to solve the integrability condition at arbitrary energy. In the original $z$ coordinates, eq.(34) takes the form

$$
\Im\left\{\left[S\left(\mathcal{K}_{, z z z} \mathcal{K}_{, z \bar{z}}+2 \mathcal{K}_{, z z \bar{z}} \mathcal{K}_{, z z}\right)+\frac{1}{2} S^{\prime}\left(\mathcal{K}_{, z z \bar{z}} \mathcal{K}_{, z}+\frac{5}{2} \mathcal{K}_{, z z} \mathcal{K}_{, z \bar{z}}\right)+\frac{1}{4} S^{\prime \prime} \mathcal{K}_{, z \bar{z}} \mathcal{K}_{, z}\right]_{, z}\right\}=0 .
$$

Substituting the ansatz (39) into eq.(49), this condition provides a second degree polynomial in $E$ :

$$
A_{2} E^{2}+A_{1} E+A_{0}=0 .
$$

The coefficients $A_{0}, A_{1}, A_{2}$ must vanish separately if the equation must hold for arbitrary energy. Therefore we obtain the three equations:

$$
\begin{aligned}
A_{2}= & \Im\left\{\left[\Lambda^{\prime \prime \prime} S+\frac{5}{4} \Lambda^{\prime \prime} S^{\prime}+\frac{1}{4}\left(\bar{z}+\Lambda^{\prime}\right) S^{\prime \prime}\right]_{, z}\right\}=0, \\
A_{1} & =\Im\left\{\left[S\left(\Psi_{, z z z}+2 \Lambda^{\prime \prime \prime} \Psi_{, z \bar{z}}+2 \Lambda^{\prime \prime} \Psi_{, z z \bar{z}}\right)\right.\right. \\
& +\frac{1}{2} S^{\prime}\left(\left(\bar{z}+\Lambda^{\prime}\right) \Psi_{, z z \bar{z}}+\frac{5}{2}\left(\Psi_{, z z}+\Lambda^{\prime \prime} \Psi_{, z \bar{z}}\right)\right) \\
& \left.\left.+\frac{1}{4} S^{\prime \prime}\left(\Psi_{, z}+\left(\bar{z}+\Lambda^{\prime}\right) \Psi_{, z \bar{z}}\right)\right]_{, z}\right\}=0, \\
A_{0}=\Im\left\{\left[S \left(\Psi_{, z z z} \Psi_{, z \bar{z}}+\right.\right.\right. & \left.\left.\left.2 \Psi_{, z z \bar{z}} \Psi_{, z z}\right)+\frac{1}{2} S^{\prime}\left(\Psi_{, z z \bar{z}} \Psi_{, z}+\frac{5}{2} \Psi_{, z \bar{z}} \Psi_{, z z}\right)+\frac{1}{4} S^{\prime \prime} \Psi_{, z \bar{z}} \Psi_{, z}\right]_{, z}\right\}=0 .
\end{aligned}
$$

It turns useful to express the system of eqs.(51 53) also in the transformed coordinates since this simplifies the computations when the $S$ function is of higher degree. Using the notation

$$
F(w)=H^{-1}(z(w))
$$

for the inverse conformal transformation, the coefficients $A_{0}, A_{1}, A_{2}$ become

$$
\begin{gathered}
A_{2}=\Im\left\{\left[F^{\prime \prime \prime} \bar{F} F^{\prime} \bar{F}^{\prime}+2\left(F^{\prime \prime}\right)^{2} \bar{F} \bar{F}^{\prime}+\Lambda^{\prime \prime \prime} F^{\prime} \bar{F}^{\prime}+\Lambda^{\prime \prime} F^{\prime \prime} \bar{F}^{\prime}\right]_{, w}\right\}, \\
A_{1}=\Im\left\{\left[\left(F^{\prime \prime \prime} \bar{F}+\Lambda^{\prime \prime \prime}\right) \Psi_{, w \bar{w}}+2\left(F^{\prime \prime} \bar{F}+\Lambda^{\prime \prime}\right) \Psi_{, w w \bar{w}}+F^{\prime} \bar{F}^{\prime} \Psi_{, w w w}+2 F^{\prime \prime} \bar{F}^{\prime} \Psi_{, w w}\right]_{, w}\right\}, \\
A_{0}=\Im\left\{\left[\Psi_{, w w w} \Psi_{, w \bar{w}}+2 \Psi_{, w w \bar{w}} \Psi_{, w w}\right]_{, w}\right\} .
\end{gathered}
$$

Applying the differential operator

$$
\frac{\partial^{2}}{\partial z \partial \bar{z}}
$$

to eq.(51), we get the condition

$$
\Im\left\{S^{\prime \prime \prime \prime}(z)\right\}=0,
$$

so that the form allowed to the $S$ function to have integrability at arbitrary energy is

$$
S(z)=a z^{4}+\beta z^{3}+\gamma z^{2}+\delta z+\epsilon, \quad a \in \mathbb{R}, \quad \beta, \gamma, \delta, \epsilon \in \mathbb{C}
$$


In [2] it has been shown that, to get arbitrary energy quadratic invariants in systems with two degrees of freedom, the function $S(z)$ must satisfy the condition

$$
\Im\left\{S_{2}^{\prime \prime}(z)\right\}=0,
$$

that is it must be a second degree polynomial with real second derivative. Together with the result (58) and the corresponding one obtained in the third-rank case [10],

$$
\Re\left\{S_{3}^{\prime \prime \prime}(z)\right\}=0,
$$

we can guess that, as a general rule, the analytic function $S(z)$ representing the conformal part of a Killing tensor of arbitrary rank $m$ is required to satisfy the condition

$$
\begin{aligned}
& \Re\left\{\left(\frac{d}{d z}\right)^{m} S(z)\right\}=0 \quad(m \text { odd }), \\
& \Im\left\{\left(\frac{d}{d z}\right)^{m} S(z)\right\}=0 \quad(m \text { even }) .
\end{aligned}
$$

As a simple illustration of the solution of the above set of eqs.(51)-(53), let us take

$$
\Lambda=0, S=b, b \in \mathbb{R} .
$$

Eq.(51) is automatically satisfied. Eq.(52) gives then the equation

$$
\Psi_{, x x x y}-\Psi_{, x y y y}=0,
$$

whose solution is

$$
\Psi_{, x y}=F_{1}(x+y)+F_{2}(x-y) .
$$

Therefore

$$
\Psi=f_{1}(x)+f_{2}(y)+f_{3}(x-y)+f_{4}(x+y) .
$$

The potential can then be written as

$$
V=v_{1}(x)+v_{2}(y)+v_{3}(x-y)+v_{4}(x+y),
$$

where $v_{i}=f_{i}^{\prime \prime} / 4, i=1,2$ and $v_{i}=f_{i}^{\prime \prime} / 2, i=3,4$. Eq.(53) now gives

$$
\begin{aligned}
& \left(v_{1}^{\prime \prime} v_{4}+2 v_{4}^{\prime \prime} v_{1}\right)+3 v_{1}^{\prime} v_{4}^{\prime}-\left(v_{1}^{\prime \prime} v_{3}+2 v_{3}^{\prime \prime} v_{1}\right)-3 v_{1}^{\prime} v_{3}^{\prime}- \\
& \left(v_{2}^{\prime \prime} v_{4}+2 v_{4}^{\prime \prime} v_{2}\right)-3 v_{2}^{\prime} v_{4}^{\prime}+\left(v_{2}^{\prime \prime} v_{3}+2 v_{3}^{\prime \prime} v_{2}\right)-3 v_{2}^{\prime} v_{3}^{\prime}=0 .
\end{aligned}
$$

This equation coincides with that reported in the review of Hietarinta [9]. Its most relevant solutions, together with the expressions of the invariant, are listed in table 2 below.

In more general cases eq.(52) is not as easily solved. The standard approach is to restrict the attention to special cases where $S(z)$ is either a homogeneous polynomial or a non-homogeneous one which is simply a power of the corresponding function in lower rank cases. Even with such restrictions, many solutions can be obtained. Moreover, in all the solutions found it has turned out that $\Lambda(z)=\lambda z^{2}$, with $\lambda$ a complex constant. In this way the coefficient

$$
z \bar{z}+2 \Re\{\Lambda(z)\}
$$

of $E$ in the Kähler potential (39) is always a Hermitian form in $z$. In the tables included in Section 6 are listed all the solutions found.

\section{$5 \quad$ Strong invariants generated by lower-order weak invariants}

In the present section we want to introduce an alternative technique to identify classes of strongly integrable systems. This approach works only in a restricted subclass of systems, but is useful to get insight into the structure of general solutions. The key point of the idea is based on the construction of a generic weak integrable system corresponding to the existence of a second-rank Killing tensor. Introducing the corresponding simplified Kähler potential in the general set of equations, we try to isolate single systems that satisfy them. The reader more interested in the general approach may wish to skip directly to section 6 with the results. 


\subsection{Quadratic invariants}

The technique devised and applied in paper [2] amounts, in short synthesis, in a conformal transformation of the form (25). In the second rank case, it turns out that it must be generated by an arbitrary holomorphic function $S_{2}(z)$ via the relation

$$
H(z)=\int \frac{d z}{\sqrt{S_{2}(z)}} .
$$

In the new coordinates $X, Y$, the Jacobi potential $G=E-V$ turns out to be of the form

$$
G(X, Y)=\frac{A(X)+B(Y)}{\left|S_{2}(X, Y)\right|},
$$

where $A$ and $B$ are arbitrary functions of their arguments, which can be thought of as the separation coordinates. The conformal factor appearing in (64) can be written as

$$
\left|S_{2}(X, Y)\right|=\sqrt{S_{2}(w) \bar{S}_{2}(\bar{w})}=F^{\prime}(w) \bar{F}^{\prime}(\bar{w}) .
$$

Moreover, we can write the transformation of the momenta in the form

$$
\begin{aligned}
& p_{X}=R p_{x}+Q p_{y}, \\
& p_{Y}=-Q p_{x}+R p_{y},
\end{aligned}
$$

where

$$
\begin{aligned}
& R=\Re\left\{F^{\prime}\right\}, \\
& Q=\Im\left\{F^{\prime}\right\} .
\end{aligned}
$$

We can therefore write the expression of the second invariant in the Jacobi gauge, as

$$
I_{S}\left(p_{X}, p_{Y}, X, Y\right)=\frac{1}{2}\left(p_{X}^{2}-p_{Y}^{2}\right)+B(Y)-A(X) .
$$

As remarked in the introduction, since to each Jacobi potential $G$ pertains a specific dynamical system, we have that the found invariant is, in general, only a weak invariant for the standard Hamiltonian, in the sense that it provides a conserved quantity only at a given value of the energy (perhaps at zero energy). In fact, the Poisson bracket of the function (68) with the separated Hamiltonian

$$
\mathcal{H}_{S}=\frac{\frac{1}{2}\left(p_{X}^{2}+p_{Y}^{2}\right)-A(X)-B(Y)}{\left|S_{2}\right|},
$$

is

$$
\left\{I_{S}, \mathcal{H}_{S}\right\}=\frac{E}{\left|S_{2}\right|}\left(p_{X}\left|S_{2}\right|_{, X}-p_{Y}\left|S_{2}\right|_{, Y}\right) .
$$

\subsection{Quartic strong invariants generated by quadratic weak invariants}

In section 4, the system of equations (51)-(53) was treated and many non trivial solutions were found. A general solution is however very difficult to find due to the complicated structure of the full system. Here we want instead to exploit the above results to generate new solutions simply starting from the assumption of the existence of a second-rank Killing tensor.

As a foreword to this approach, let us make the following remark. Let us suppose to have made the choice of the $S_{2}$ function generating the conformal transformation which gives the second invariant (68) satisfying equation $(70)$. Denoting by $\mathcal{H}_{0}$ the numerator appearing in the expression (69) of the Hamiltonian, the Poisson bracket of a generic higher-order invariant with $\mathcal{H}_{S}$ can be written as

$$
\begin{aligned}
\left\{I_{J}, \mathcal{H}_{S}\right\} & =\frac{1}{\left|S_{2}\right|}\left\{I_{J}, \mathcal{H}_{0}\right\}-\frac{\mathcal{H}_{0}}{\left|S_{2}\right|^{2}}\left\{I_{J},\left|S_{2}\right|\right\} \\
& =\frac{\left\{I_{J}, \mathcal{H}_{0}\right\}-E\left\{I_{J},\left|S_{2}\right|\right\}}{\left|S_{2}\right|} .
\end{aligned}
$$


In the case of a second-order invariant in the standard form of (68), this reduces to 70 ). On the other hand, let us introduce the null Hamiltonian

$$
\mathcal{H}_{\mathcal{N}}=\left(\mathcal{H}_{S}-E\right)\left|S_{2}\right|=\mathcal{H}_{0}-E\left|S_{2}\right|
$$

The Poisson bracket of the invariant with $\mathcal{H}_{\mathcal{N}}$ is

$$
\left\{I_{J}, \mathcal{H}_{\mathcal{N}}\right\}=\left\{I_{J}, \mathcal{H}_{0}\right\}-E\left\{I_{J},\left|S_{2}\right|\right\}
$$

Since $\left|S_{2}\right|$ is a non-null positive function everywhere, eqs.(71) and (73) are equivalent for what concernes the conservation of $I_{J}$. The conceptual difference relies on the fact that, whereas eq.(73) expresses the conservation of $I_{J}$ in the dynamics provided by $\mathcal{H}_{\mathcal{N}}$ at zero "energy", eq.(71) expresses the conservation of $I_{J}$ in the dynamics provided by $\mathcal{H}_{S}$ at arbitrary energy. Note that the true energy $E$ enters into $\mathcal{H}_{\mathcal{N}}$ as an arbitrary parameter.

In the light of this argument, the procedure to look for new integrable systems is the following: regard the null Hamiltonian, written in the form

$$
\mathcal{H}_{\mathcal{N}}=\frac{1}{2}\left(p_{X}^{2}+p_{Y}^{2}\right)-\tilde{G}(X, Y)
$$

where

$$
\tilde{G}(X, Y)=A(X)+B(Y)+E\left|S_{2}(X, Y)\right|
$$

as the system in which to find a new conserved quantity at zero energy. If the search of this new conserved quantity is successful, then it is a strongly conserved quantity for the original Hamiltonian $\mathcal{H}_{S}$, which is therefore integrable at arbitrary energy. Going backwards with respect to the previous approach, we take the potential of eq. 75 ) and use it to solve the system (33). Once we have the solution, we can write the integrability condition (34) in the form

$$
\Im\left\{R_{, w w} G+3 R_{, w} G_{, w}+2 R G_{, w w}\right\}=0 .
$$

Note that in the remaining part of this section we suppress the "tilde" over $\tilde{G}$ and $\tilde{R}$ to simplify the notation. The solution of this equation provides the explicit form of the functions $A(w+\bar{w})$ and $B(w-\bar{w})$ that allow for the integrability of the system. Finally, the integration of the equations for the trace (38) completes the solution.

In the development of this approach, it can be useful to come back to the separating real variables. Equations (33) involving the Kähler potential therefore become:

$$
\begin{aligned}
G(X, Y) & =\frac{1}{4}\left(\mathcal{K}_{, X X}+\mathcal{K}_{, Y Y}\right), \\
R(X, Y) & =\frac{1}{2}\left(\mathcal{K}_{, Y Y}-\mathcal{K}_{, X X}\right)+i \mathcal{K}_{, X Y},
\end{aligned}
$$

and the integrability condition (76) can be written in the form

$$
\begin{aligned}
& {\left[(\Im\{R\})_{, X X}-(\Im\{R\})_{, Y Y}-2(\Re\{R\}), X Y\right] G+} \\
& 3\left[\left((\Im\{R\})_{, X}-(\Re\{R\})_{, Y}\right) G_{, X}-\left((\Re\{R\})_{, X}+(\Im\{R\}), Y\right) G_{, Y}\right]+ \\
& 2\left[\Im\{R\}\left(G, X X-G_{, Y Y}\right)-2 \Re\{R\} G_{, X Y}\right]=0 .
\end{aligned}
$$

The system (38) finally becomes:

$$
\begin{aligned}
K_{, X} & \left.=-\frac{4}{3}\left((\Re\{R\})_{, X}+(\Im\{R\})_{, Y}\right)\right) G-\frac{8}{3}\left(\Re\{R\} G_{, X}+\Im\{R\} G_{, Y}\right), \\
K_{, Y} & =-\frac{4}{3}\left((\Im\{R\})_{, X}-(\Re\{R\})_{, Y}\right) G-\frac{8}{3}\left(\Im\{R\} G_{, X}-\Re\{R\} G_{, Y}\right) .
\end{aligned}
$$


To solve system (33), we need only consider the contribution to the potential (75) coming from the conformal factor:

$$
G_{C}=E\left|S_{2}(X, Y)\right| \text {. }
$$

Recalling the relation (63) between the conformal transformation and the $S_{2}$ function in the quadratic case and the definition of the inverse transformation (54), the above source term can also be written as

$$
G_{C}=E \sqrt{S_{2}(w) \bar{S}_{2}(\bar{w})}=E F^{\prime}(w) \bar{F}^{\prime}(\bar{w}) .
$$

The solutions of system (33) for this term are:

$$
\begin{aligned}
& \mathcal{K}_{C}=E(F(w) \bar{F}(\bar{w})+2 \Re\{\Lambda(w)\}), \\
& R_{C}=-2 E\left(F^{\prime \prime}(w) \bar{F}(\bar{w})+\Lambda^{\prime \prime}(w)\right)
\end{aligned}
$$

so that the complete solution is therefore

$$
\begin{aligned}
& \mathcal{K}=4 \int\left[\int A d X\right] d X+4 \int\left[\int B d Y\right] d Y+E(F(w) \bar{F}(\bar{w})+2 \Re\{\Lambda(w)\}), \\
& R=2(B(Y)-A(X))-2 E\left(F^{\prime \prime}(w) \bar{F}(\bar{w})+\Lambda^{\prime \prime}(w)\right)
\end{aligned}
$$

This solution is subject to the integrability condition $(76)$. In analogy with the general approach, substituting in it the solution (83) we get a second degree polynomial in $E$ of the form (50). This equation must be satisfied for every value of the arbitrary parameter $E$, so that each coefficient must separately vanish. The three coefficients are in this case:

$$
\begin{aligned}
A_{2}= & -4 i \Im\left\{\left(F^{\prime \prime \prime \prime} \bar{F}+\Lambda^{\prime \prime \prime \prime}\right) F^{\prime} \bar{F}^{\prime}+5 F^{\prime \prime \prime} F^{\prime \prime} \bar{F}^{\prime} \bar{F}+3 \Lambda^{\prime \prime \prime} F^{\prime \prime} \bar{F}^{\prime}+2 \Lambda^{\prime \prime} F^{\prime \prime \prime} \bar{F}^{\prime}\right\}, \\
A_{1}= & 4 i \Im\left\{3\left(B_{, w}-A_{, w}\right) F^{\prime \prime} \bar{F}^{\prime}-3\left(A_{, w}+B_{, w}\right)\left(F^{\prime \prime \prime} \bar{F}+\Lambda^{\prime \prime \prime}\right)+2(B-A) F^{\prime \prime \prime} \bar{F}^{\prime}\right. \\
& \left.-2\left(A_{, w w}+B_{, w w}\right)\left(F^{\prime \prime} \bar{F}+\Lambda^{\prime \prime}\right)-(A+B) F^{\prime \prime \prime \prime} \bar{F}\right\}, \\
A_{0}= & 2 i \Im\left\{6\left(B_{, w}-A_{, w}\right)\left(B_{, w}+A_{, w}\right)+4(B-A)\left(A_{, w w}+B_{, w w}\right)\right\} .
\end{aligned}
$$

From the properties of the functions $A$ and $B, A_{0}$ is identically zero. Limiting ourselves to the simplest case $\Lambda=0$, the condition that $A_{2}$ vanishes turns out to be

$$
\Im\left\{\left(F^{\prime \prime \prime \prime} F^{\prime}+5 F^{\prime \prime \prime} F^{\prime \prime}\right) \bar{F} \bar{F}^{\prime}\right\}=0,
$$

so that it is necessary and sufficient that

$$
F^{\prime \prime \prime \prime} F^{\prime}+5 F^{\prime \prime \prime} F^{\prime \prime}=6 a F F^{\prime}, \quad a \in \mathbb{R} .
$$

The general solution is

$$
\left(F^{\prime}\right)^{4}=a F^{4}+\gamma F^{2}+\delta F+\epsilon
$$

where $\beta, \gamma, \delta, \epsilon$ are complex constants. We see that solution (87) is a subset of the general result (58) so that we can state that the simplified approach presented here consists in choosing a function in the set

$$
S_{2}(z)=\sqrt{a z^{4}+\gamma z^{2}+\delta z+\epsilon}
$$

The equation $A_{1}=0$ then picks the functions $A$ and $B$ that are compatible with strong integrability. The practical advantage with respect to the application of the general technique of section 4 is that equation $A_{0}=0$ is automatically satisfied. 


\subsection{Class $i z^{2}$ systems}

To illustrate the simplified approach, in this subsection we present a class of simple systems which are strongly integrable with a quartic invariant and, at zero energy, are separable with a quadratic invariant.

Consider then the function

$$
S_{2}(z)=i z^{2}
$$

In the solution 87 it corresponds to the choice

$$
a=-1, \gamma=\delta=\epsilon=0 .
$$

This is the simplest polynomial form which does not satisfy the constraint (60) and therefore gives a potential which is not automatically integrable at arbitrary energy with a quadratic second invariant. The corresponding conformal transformation is given by

$$
w=H(z)=\frac{1-i}{\sqrt{2}} \ln z .
$$

Using polar coordinates, so that

$$
\begin{aligned}
& z=x+i y=r \exp (i \theta), \\
& r=\sqrt{x^{2}+y^{2}}, \quad \theta=\arctan (y / x),
\end{aligned}
$$

in terms of real variables, we get

$$
\begin{aligned}
& X=\frac{1}{\sqrt{2}}(\theta+\ln r), \\
& Y=\frac{1}{\sqrt{2}}(\theta-\ln r) .
\end{aligned}
$$

We point out, in passing, that that in [2], in equations (102-104) corresponding to eq. (93) above, there is a misprint and the coordinate $r$ must be substituted with its natural logarithm. It follows that the potential given by

$$
V(\theta, r)=-\frac{A(\theta+\ln r)+B(\theta-\ln r)}{r^{2}}
$$

is integrable at zero energy for arbitrary functions $A$ and $B$. The common factor $r^{-2}$ is due to the fact that, from the choice of eq. (89), we have

$$
|S(X, Y)|=r^{2}=\mathrm{e}^{\sqrt{2}(X-Y)} .
$$

The solutions (83) are

$$
\mathcal{K}=4 \int\left[\int A d X\right] d X+4 \int\left[\int B d Y\right] d Y+E \mathrm{e}^{\sqrt{2}(X-Y)} .
$$

and

$$
R=2\left((B(Y)-A(X))-2 i E \mathrm{e}^{\sqrt{2}(X-Y)} .\right.
$$

The integrability condition (78) becomes

$$
4 E \mathrm{e}^{\sqrt{2}(X-Y)}\left[A^{\prime \prime}-B^{\prime \prime}+3 \sqrt{2}\left(A^{\prime}+B^{\prime}\right)+4(A-B)\right]=0 .
$$

This equation splits into

$$
\begin{aligned}
& A^{\prime \prime}+3 \sqrt{2} A^{\prime}+4 A=0, \\
& B^{\prime \prime}-3 \sqrt{2} B^{\prime}+4 B=0,
\end{aligned}
$$

with solutions:

$$
\begin{aligned}
& A(X)=a_{1} \mathrm{e}^{-\sqrt{2} X}+a_{2} \mathrm{e}^{-2 \sqrt{2} X}, \\
& B(Y)=b_{1} \mathrm{e}^{\sqrt{2} Y}+b_{2} \mathrm{e}^{2 \sqrt{2} Y} .
\end{aligned}
$$


The potential

$$
V(r, \theta)=-\frac{a_{1} \mathrm{e}^{-\theta}+b_{1} \mathrm{e}^{\theta}}{r^{3}}-\frac{a_{2} \mathrm{e}^{-2 \theta}+b_{2} \mathrm{e}^{2 \theta}}{r^{4}}
$$

ie therefore integrable. As a simple concrete example, let us take

$$
a_{1}=b_{1}=0, a_{2}=b_{2}=-\frac{1}{2},
$$

so that the potential is

$$
V=\frac{\mathrm{e}^{2 \theta}+\mathrm{e}^{-2 \theta}}{2 r^{4}}
$$

which is a known result mentioned in the review by Hietarinta. The second invariant has the form

$$
I=p_{\theta}^{4}+\operatorname{ch} 2 \theta p_{r}^{2}+2 \frac{\operatorname{sh} 2 \theta}{r} p_{r} p_{\theta}+3 \frac{\operatorname{ch} 2 \theta}{r^{2}} p_{\theta}^{2}+2 \frac{2-\operatorname{sh} 2 \theta}{r^{4}} .
$$

\section{Classification of Hamiltonians admitting a quartic invariant at arbitrary energy}

In the tables included in this section we present all the solutions we have found of systems admitting an independent invariant quartic in the momenta. Some of them are new. For all cases we present the potential $V$ (up to linear transformations of the coordinates and a linear rescaling of the potential itself) as well as the quartic invariant $I$, thereby making it possible to directly compare our results with Hietarinta's classification of 1987 [9]. For the cases which are superintegrable we also indicate, using the notation of [9], which of the real quadratic cases (1), (2), (4) or (7) a given system belongs to.

A peculiar property of our solutions is that all but one have a conformal factor generated by function $S$ of even degree in $z$, with real coefficients. As a consequence of this, the solutions obtained can be expressed in terms of the coordinate systems of separable potentials. In fact, since from (28) and (54), the transformation is

$$
H(z)=F^{-1}(w(z))=\int d z\left[S_{4}(z)\right]^{-1 / 4},
$$

with $S$ of the form

$$
S_{4}(z)= \pm\left(a z^{2}+\beta z+\gamma\right), \quad a \in \mathbb{R}, \quad \beta, \gamma \in \mathbb{C},
$$

we are actually taken back to the coordinate transformations to separable variables given by an $S_{2}$ function satisfying the condition [60, for which the transformation is of the form [2]

$$
H(z)=\int d z\left[S_{2}(z)\right]^{-1 / 2} .
$$

As an exception to this situation, we have also found a solution with $S_{4}$ of third degree in $z$, by the method of the coupling constant metamorphosis (see [9]) which is described in Section 6.2 below.

\subsection{Systems for which $S(z)$ is of even degree}

The simplest case is that with $S_{4}$ of degree zero. This case corresponds simply to rotations and translations of the Cartesian coordinates. In table 1 we list the new solutions obtained and, for comparison, the quartic integrable potential of [13. In table 2 the already known solutions of this class are listed together with the proper references. The first sequence of items correspond to the simple solution sketched at the end of section 4. All solutions are generated by $S_{4}= \pm 1$ : actually, there is nothing fundamental in the choice of sign which has been made only for sake of comparison with already known results.

We then have some solutions with $S_{4}$ of degree two and four in $z$. In table 3 , two new interesting classes of integrable systems are presented with $S_{4}$ of degree two. They are more conveniently treated using the 
parabolic coordinates provided by the conformal transformation generated by $S_{4}=16 z^{2}$. In table 5 , a class of integrable systems generated by $S_{4}=-z^{4}$ is presented, generalizing the separability in polar coordinates. Here, the choice of sign is dictated by the transformation used in the simplified approach of section 5.3 above, but to the same system one can also arrive with the general method using the "proper" transformation given by $S_{4}=z^{4}$.

\subsection{A system for which $S(z)$ is of degree three}

In order to describe how we get this new solution, we briefly recall the method based on the coupling constant metamorphosis [9]. We introduce a null Hamiltonian which, in analogy with eq.(72), is defined as

$$
\mathcal{H}_{\mathcal{N}}=(\mathcal{H}-E)\left|S_{4}\right|^{2} .
$$

The second case of table 3 has a null Hamiltonian which, if expressed in the new coordinates, has the form

$$
\mathcal{H}_{\mathcal{N}}=\frac{1}{2}\left(p_{X}^{2}+p_{Y}^{2}\right)-A(X)-B(Y)+4 C\left(X^{2}+Y^{2}\right)^{3}-4 E\left(X^{2}+Y^{2}\right) .
$$

Then, if the term $\left(X^{2}+Y^{2}\right)^{3}$ can be identified with the conformal factor associated to a new conformal transformation, we may interpret $C$ as a new energy and $E$ as a new coupling constant. We get a new potential which is strongly integrable with an invariant of the same nature as before. This is the process known as coupling constant metamorphosis.

In the present instance, we have that the transformation must be still of the form (105) or, equivalently,

$$
F^{\prime}(w)=S_{4}^{1 / 4}
$$

where, for simplicity, we still denote the new variable as $w=X+i Y$, so that $|w|^{2}=X^{2}+Y^{2}$. Since the conformal factor is assumed to be $\left(X^{2}+Y^{2}\right)^{3}$, we get

$$
\left|F^{\prime}\right|^{2} \sim|w|^{6}
$$

Therefore, $F(w)$ must be of the order $w^{4}$, so that

$$
w=H(z) \sim z^{1 / 4} .
$$

From 105) we get,

$$
H^{\prime}(z) \sim z^{-3 / 4}
$$

so that, finally,

$$
S_{4}(z) \sim z^{3} .
$$

We end up with a solution of third degree for $S$ which had escaped a previous direct analysis. The new integrable Hamiltonian is

$$
\begin{aligned}
\mathcal{H}= & \frac{1}{2}\left(p_{r}^{2}+\frac{p_{\theta}^{2}}{r^{2}}\right)+\frac{\mu}{r}+ \\
& \frac{a}{r^{2}\left(1+\cos \frac{\theta}{2}\right)}+\frac{b}{r^{2}\left(1-\cos \frac{\theta}{2}\right)}+\frac{c}{r^{3}\left(1+\cos \frac{\theta}{2}\right)^{3}}+\frac{d}{r^{3}\left(1-\cos \frac{\theta}{2}\right)^{3}}=\tilde{E},
\end{aligned}
$$

where $\tilde{E}=C$ is the new energy and $\mu=-E$ is the new coupling constant. Table 4 reports the form of the coordinate transformation and the expression of the second invariant. 


$$
\begin{aligned}
& S(z)=1 \\
& \mathcal{K}=2 E x^{2}-\frac{4}{3} x^{4}-2 b x y^{2}-\frac{1}{3} y^{4}+4 c \ln |y| \\
& V=4 x^{2}+y^{2}+b x+c y^{-2} \\
& I=p_{x}^{4}+2 p_{x}^{2} p_{y}^{2}+4 V p_{x}^{2}+4 b y p_{x} p_{y}+16 x^{2} p_{y}^{2}+2\left(2 x^{2}+y^{2}\right)(b+4 x)^{2}+32 c x^{2} y^{-2}
\end{aligned}
$$

Superintegrable, case (4) and (7)

\begin{tabular}{l}
\hline \hline$S(z)=1$ \\
$\mathcal{K}=2 E x^{2}-9 y^{4 / 3}(b x+c)-\frac{3}{2} a y^{4}-\frac{9}{10} d y^{8 / 3}-\frac{8}{3} a x^{4}$ \\
$V=\frac{1}{2} a\left(16 x^{2}+9 y^{2}\right)+(b x+c) y^{-2 / 3}+d y^{2 / 3}$ \\
$I=p_{x}^{4}+2 p_{x}^{2} p_{y}^{2}+4 V p_{x}^{2}+12 b y^{1 / 3} p_{x} p_{y}+32 a x^{2} p_{y}^{2}+64 a x^{2} V$ \\
$\quad+16 b d x-256 a^{2} x^{4}+18 b^{2} y^{2 / 3}+144 a b x y^{4 / 3}$ \\
\hline \hline$S(z)=-1$ \\
$\mathcal{K}=2 E x^{2}-2 x^{4} y^{2}-\frac{1}{3} a x^{4} y-\frac{a^{2}}{12}\left(\frac{x^{4}}{18}+y^{4}\right)-\frac{16}{15} y^{6}-\frac{8}{15} a y^{5}+4 d \ln |x|$ \\
$V=x^{4}+6 x^{2} y^{2}+8 y^{4}+a\left(\frac{8}{3} y^{3}+x^{2} y\right)+\frac{a^{2}}{72}\left(x^{2}+16 y^{2}\right)+\frac{d}{x^{2}}$, \\
$I=p_{x}^{4}+4 p_{x}^{2}\left(x^{4}+6 x^{2} y^{2}+a x^{2} y+\frac{1}{72} a^{2} x^{2}+\frac{d}{x^{2}}\right)-\left(\frac{4}{3} a x^{3}+16 x^{3} y\right) p_{x} p_{y}$ \\
$\quad+4 x^{4} p_{y}^{2}+4\left(2 d x^{2}+x^{8}+d^{2} x^{-4}+\frac{2}{3} a d y+4 d y^{2}\right)+$ \\
$\quad \frac{x^{6}}{9}\left(144 y^{2}+24 a y-a^{2}\right)+\frac{x^{4}}{1296}\left(144 y^{2}+24 a y-a^{2}\right)^{2}$ \\
\hline \hline$S(z)=-1$ \\
$\mathcal{K}=2 E x^{2}-2 x^{4} y^{2}-\frac{16}{15} y^{6}-\frac{1}{3} b x^{4}-\frac{4}{3} b y^{4}+4 d \ln |x|-\frac{c}{5} y^{-4}+4 m \ln |y|$ \\
$V=x^{4}+6 x^{2} y^{2}+8 y^{4}+b\left(x^{2}+4 y^{2}\right)+\frac{d}{x^{2}}+\frac{m}{y^{2}}+\frac{c}{x^{6}},(\mathrm{Grammaticos}$ et al. 1984$)$ \\
$I=p_{x}^{4}+4 p_{x}^{2}\left(x^{4}+6 x^{2} y^{2}+b x^{2}+\frac{d}{x^{2}}+\frac{c}{x^{6}}\right)-16 x^{3} y p_{x} p_{y}+4 x^{4} p_{y}^{2}$ \\
$\quad+4\left[12 c y^{2} x^{-4}+2 d x^{2}+x^{8}+2 b x^{6}+b^{2} x^{4}+2 c x^{-2}+2 c d x^{-8}+c^{2} x^{-12}+\left(d^{2}+2 b c\right) x^{-4}\right.$ \\
$\left.\quad+4 x^{6} y^{2}+2 m x^{4} y^{-2}+4 b x^{4} y^{2}+4 x^{4} y^{4}+4 d y^{2}\right]$ \\
\hline
\end{tabular}

Table 1: Systems for which $S(z)$ is of zero degree. 


\section{Conclusions}

In this work we have explored the set of natural 2-dimensional Hamiltonian systems admitting a second invariant which is a polynomial in the momenta of degree four. The approach is based on the Jacobi geometrization described in [2], where the linear and quadratic cases were also explored, and in [10], where the cubic cases were treated. The approach allows a unified treatment of integrability at fixed and arbitrary energy, even if here we have limited ourselves to obtaining the list, as complete as possible, of strongly integrable systems. There are several areas in which this approach can be still useful. The most natural seems that of looking for systems admitting higher order invariants of which there exist only a few known examples.

Moreover, other properties of invariants up to degree four are still worth of analysis. In [14] we have examined the properties of weakly integrable systems with quadratic second invariants and found interesting behaviours both in the integrable regime and in the generic non-integrable one. One may wonder about the information higher order weak invariants can give on the dynamics of non-integrable systems. It is reasonable to believe that increasing the order can improve the reliability of these functions when interpreting them as effective invariants approximately conserved in regular portions of phase-space. It would also be interesting

to look for other examples of quartic invariants which correspond to lower rank Killing tensors on fixed energy surfaces.

\section{References}

[1] V. N. Kolokol'tsov, Math. USSR Izvestiya 21, 291 (1983).

[2] K. Rosquist and G. Pucacco, J. Phys. A 28, 3235 (1995).

[3] S. Benenti, J. Math. Phys. 38, 6578 (1997).

[4] V. I. Arnold, Mathematical methods of classical mechanics (Springer-Verlag, New York, USA, 1978).

[5] C. Lanczos, The variational principles of mechanics (Dover, New York, USA, 1986).

[6] G. Darboux, Archives Néerlandaises (ii) VI, 371 (1901).

[7] L. S. Hall, Physica 8D, 90 (1983).

[8] W. Sarlet, P. G. L. Leach and F. Cantrijn, Physica 17D, 87 (1985)

[9] J. Hietarinta, Phys. Rep. 147, 87 (1987).

[10] M. Karlovini and K. Rosquist, J. Math. Phys. 41, 370 (2000)

[11] L. Samuelsson, Master's Thesis, Department of Physics, Stockholm University (1998), available at http://www.fop.physto.se/exjobb/exjobb.htm

[12] V. V. Kozlov Symmetries, Topology and Resonances in Hamiltonian Mechanics (Berlin: Springer, 1991).

[13] B. Grammaticos, B. Dorizzi, A. Ramani, J. Math. Phys. 25, 3470 (1984)

[14] G. Pucacco and K. Rosquist, (2001), submitted 


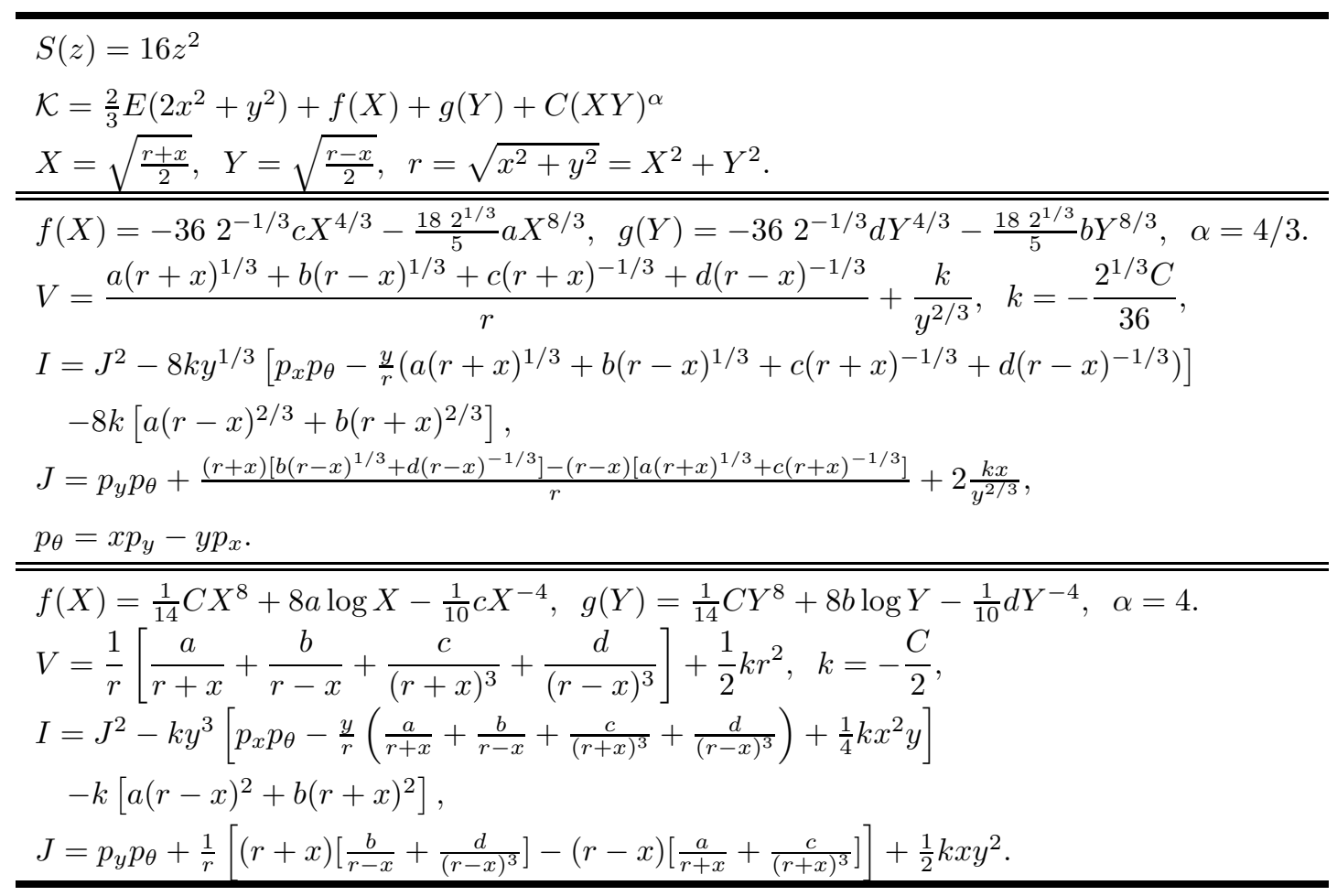

Table 3: Systems for which $S(z)$ is of second degree. 


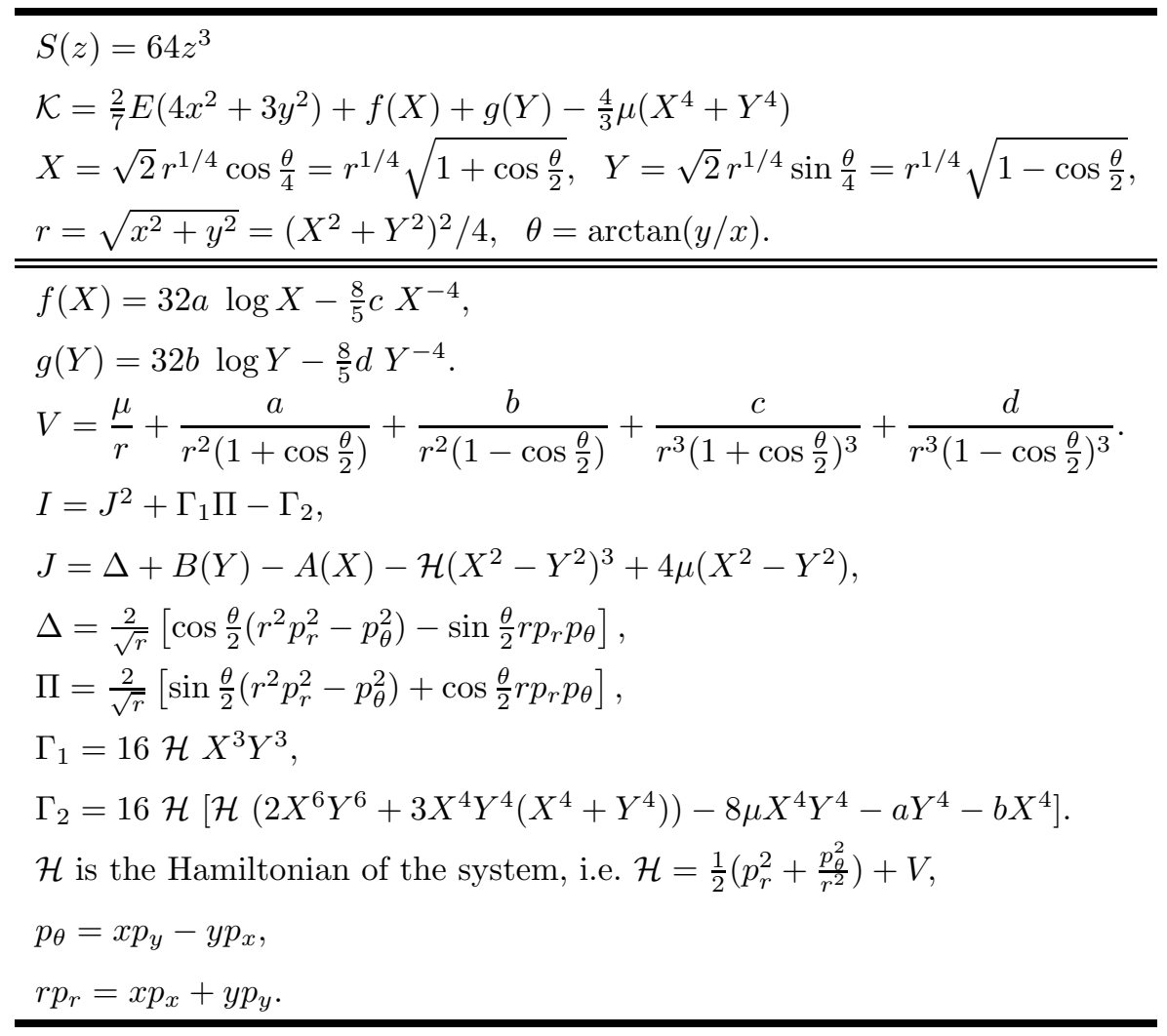

Table 4: Systems for which $S(z)$ is of third degree.

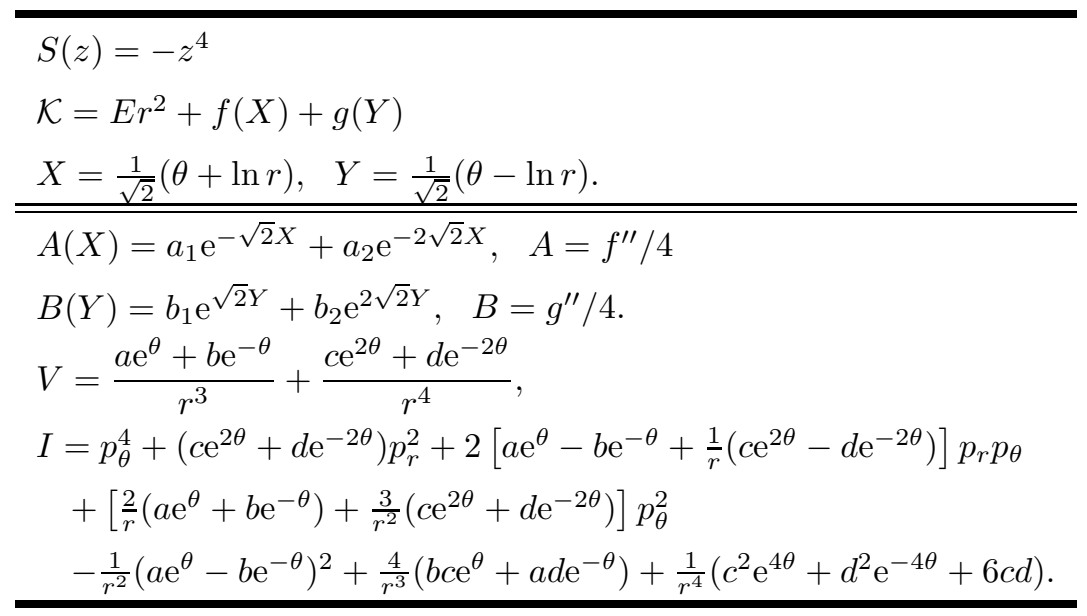

Table 5: Systems for which $S(z)$ is of fourth degree. 